\title{
Rechtsmedizin
}

Erratum

Rechtsmedizin 2021 $31: 110$

https://doi.org/10.1007/s00194-020-00450-9

Online publiziert: 11 . Dezember 2020

(c) Springer Medizin Verlag $\mathrm{GmbH}$, ein Teil von Springer Nature 2020

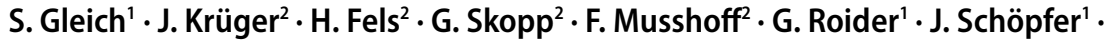
M. Graw' $\cdot$ C. Wiedfeld ${ }^{2}$

'Institut für Rechtsmedizin, LMU München, München, Deutschland

${ }^{2}$ Forensisch Toxikologisches Centrum (FTC), München, Deutschland

\section{Erratum zu: Medikamente als freiheitsentziehende Maßnahme in stationären Pflegeeinrichtungen? Eine kritische Analyse}

\section{Erratum zu:}

Rechtsmedizin 2020

https://doi.org/10.1007/s00194-020-004

40-x

Leider kam es bei diesem Beitrag zu einem Fehler in den Affiliations. Das Forensisch Toxikologische Centrum (FTC), München, Deutschland musste ergänzt werden. Die korrekte Zugehörigkeit der jeweiligen Autoren finden Sie oben, der Originalbeitrag wurde entsprechend korrigiert.

Wir bitten, dies zu entschuldigen.

\section{Korrespondenzadresse}

PD Dr. med. habil. S. Gleich

Institut für Rechtsmedizin, LMU München

Nußbaumstr. 26, 80336 München, Deutschland sabine.gleich@muenchen.de 